CPB Memorandum

CPB Netherlands Bureau for Economic Policy Analysis

\begin{tabular}{|c|c|}
\hline Sector(s) & : Labour Market and Welfare State \\
\hline Unit(s)/Project & NETSPAR project \\
\hline Author(s) & Nick Draper \\
\hline Number & 196 \\
\hline Date & 2 April 2008 \\
\hline
\end{tabular}

\title{
Prudence and Robustness as Explanations for Precautionary Savings; an Evaluation
}

This paper evaluates approximation methods to make manageable the numerical solution of overlapping generation models with aggregate risk. The paper starts with a model in which households maximize expected utility over their life cycle. Instantaneous utility is characterized by constant relative risk aversion. Prudence, a characteristic of the utility function, leads to precautionary saving. The first-order conditions include expectations. One source of uncertainty is not prohibitive for numerical integration of the expectation term. Because of its accuracy numerical integration results are used as a bench mark. Taylor series approximations can lead to the same results dependent on the linearization point. A linear quadratic approximation of the household model is evaluated subsequently. Alternatively, precautionary saving effects can be the result of robust decision making. This approach leads to linear policy functions and gives a rather good approximation of the bench mark model, although not as good as the Taylor series approximation.

JEL code: E21, D81, C61

Keywords: Precautionary saving, Robustness, Prudence

Thanks are due to Bram Edens, who contributed to section 2 of this document, to Peter Broer and Ed Westerhout for their valuable comments and to André Nibbelink and Richard Rosenbrand for their research assistance. 



\section{Contents}

\begin{tabular}{lll}
\hline 1 & Introduction & 2
\end{tabular}

\begin{tabular}{|lll}
2 & Prudence with risk as explanation for precautionary saving & 4
\end{tabular}

2.1 Introduction 4

\begin{tabular}{lll}
\hline 2.2 & Household decision problem & 4
\end{tabular}

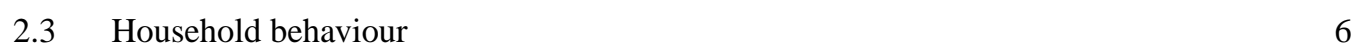

$\begin{array}{lll}2.4 \text { Calibration } & 7\end{array}$

$\begin{array}{lll}2.5 & \text { Simulations } & 7\end{array}$

\begin{tabular}{|lll}
3 & Taylor approximation of the prudence model & 9
\end{tabular}

$\begin{array}{lll}3.1 & \text { Approximating household behaviour } & 9\end{array}$

$\begin{array}{lll}3.2 \text { Simulations } & 9\end{array}$

$\begin{array}{lll}4 & \text { Robustness as explanation for precautionary saving } & 10\end{array}$

$\begin{array}{lll}4.1 \quad \text { Introduction } & 10\end{array}$

$4.2 \quad$ A linear quadratic model of the household decision 11

$\begin{array}{lll}4.3 & \text { A digression on robust control } & 13\end{array}$

\begin{tabular}{lll}
\hline 4.4 & Household behaviour & 14
\end{tabular}

\begin{tabular}{|lll}
\hline 4.5 & Simulations using a robust linear regulator & 14
\end{tabular}

\begin{tabular}{lll}
\hline 5 & Summary & 17
\end{tabular}

\begin{tabular}{lll}
\hline A & Symbols main text & 19
\end{tabular}

\begin{tabular}{lll}
\hline B & Derivations section 2 and 3 & 21
\end{tabular}

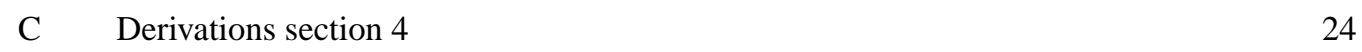

\begin{tabular}{lll}
\hline C.1 Linear quadratic approximation & 24
\end{tabular}

\begin{tabular}{lll}
\hline C.2 & Derivations robust control & 26
\end{tabular}

\begin{tabular}{lr}
\hline References & 28
\end{tabular} 
Introduction

As part of sub-project 3 of the Netspar theme ("Risk sharing in pension schemes in the presence of economic and demographic risks: applied stochastic modelling"), we want to compare pure DB schemes with pure (collective) DC schemes and with hybrid schemes that combine DB and DC elements. We want to focus on how schemes affect households directly. Therefore, we compare the different schemes in terms of premiums, benefits and net profits (benefits minus premiums, where the measurement is done in present-value terms). We do this for a large number of scenarios, allowing us to compare the pension schemes for typical scenarios and for large sets of scenarios on the basis of means and variances. We also want to account for economic behaviour, in particular saving and investment behaviour, as it would be difficult to motivate that large-scale reforms of the pension scheme would not induce any behavioural reaction on part of households.

Models that contain overlapping generations of forward-looking households and that feature aggregate uncertainty are often quite difficult to solve numerically however. Simplification, for example through linearization, could then be beneficial, if the approximation errors involved are relatively small. Therefore, it is an interesting exercise to compare different numerical approaches using a model that is so stylized that solving it numerically does not present problems. The aim of such an exercise is then to highlight the benefits (a.o., computer time) and costs (computational errors) attached to simplifying the original model.

This is what this paper does. It takes a standard life cycle model with a consumption-saving decision, a portfolio-allocation decision (two assets, of which one is risky), microeconomic uncertainty regarding the length of life, and aggregate uncertainty regarding the rate of return on equity (the risky asset). Labour productivity is non-stochastic, leaving us in a setting of complete markets. Labour supply is taken exogenous. We then first solve this model numerically. Second, we approximate the model, adopting two different approaches. The first approach derives first-order conditions for optimal household behaviour and approximates relations based on these first-order conditions. This approach leads to precautionary saving as the third derivative of the felicity function is positive. The second approach approximates the original problem with a quadratic utility function and linear restrictions. The second approach needs robust decision making to account for precautionary saving.

More saving due to increasing variability of exogenous random shocks is called precautionary saving (Kimball (1990)). Two competitive theories can explain precautionary saving. The prudence theory states that precautionary saving occurs due to prudence (convexity of the marginal utility function) with risk. The prudence with risk theory is the more traditional explanation of economic theory for precautionary saving. Alternatively, the distrust theory does 
not make use of convexity of the marginal utility function (quadratic utility is allowed) but introduces distrust of households' knowledge about the real world. This distrust theory makes use of robust control and has quite recently been developed (Hansen and Sargent (2007)).

This paper formulates and compares two household models each based on one of the two explanations of precautionary saving. The traditional, prudence model uses utility functions with positive third derivatives. For instance CRRA instantaneous utility functions have this property. The distrust model uses a linear quadratic instantaneous utility function and has linear restrictions. Without distrust standard linear quadratic Gaussian control (LQG) could be applied. Features of LQG are:

1. it can be used as a second-order approximation of nonlinear quadratic control problems

2. the optimal decision rule is linear and becomes time-invariant in an infinite horizon setting

3. the optimal decision rule is invariant to the magnitude of the stochastic disturbances in the model (certainty equivalence).

The first characteristic links the method to theoretical practise to linearize complicated models to make them empirically manageable. The second characteristic holds only partially in overlapping generation models. A characteristic of overlapping generation models is the finite horizon of households. This characteristic leads to age-dependent linear optimal decision rules of households, which are not time-dependent. The third characteristic of LQG reduces the applicability for consumption-saving decisions. Standard LQG predicts savings independent of the degree of uncertainty. Jacobson (1973) and Whittle $(1981,1990)$ sought to retain the good features of the LQG problem (linearity and time invariance) while incorporating some role for the variance of the disturbances in the optimal decision rule. Their approach is known as robust control. Hansen and Sargent (2007) modified robust control methods to make them useful for economic applications. Robust control introduces distrust about the model specification. The actual developments may lay in an environment of the specified model. This leads to utility maximization given a possible worst case scenario which is constructed using an entropy measure for possible misspecification. Robust control makes the decision rule robust against possible misspecification.

Apart from comparing both explanations for precautionary saving, this paper contributes to the development of robust control methods. Hansen and Sargent (2007) and Tallarini (2000) use robust control in real business cycle models with one representative household with an infinite time horizon leading to a time-invariant optimal decision rule. The model discussed here is developed for a household with a finite life expectancy. The optimal decision rule becomes age-dependent.

The linear recursive character of the robust control model is convenient. It makes stochastic 
simulation within an overlapping generation model to study insurance against macro-economic risks easier to implement than the prudence approach.

\section{$2 \quad$ Prudence with risk as explanation for precautionary saving}

\section{$2.1 \quad$ Introduction}

This section starts in 2.2 with formulating a household problem based on CRRA instantaneous utility. Households save due to a precautionary saving motive. Only one macroeconomic risk is distinguished: equity income is uncertain. So, labour income is nonstochastic. Household behaviour comes into discussion in 2.3 Subsection 2.4 discusses the calibration of the model. The simulation results are presented in 2.5

\subsection{Household decision problem}

An individual of age $j$ maximizes his expected remaining lifetime utility $U$, which depends on per-period utility $u$ and on the subjective discount factor $d_{s}$. Expectations have to be formed for two reasons. First the returns on assets are uncertain. Second, length of life is uncertain, so it is assumed that individuals weigh their future per-period utility with survival probabilities. The lifetime utility function reads as

$$
\begin{aligned}
U_{j} & =E_{j} \sum_{i=j}^{j e} u_{i} d_{s i} \text { with } \\
d_{S}(i) & =\delta^{-(i-j)} \prod_{l=j}^{i-1} \zeta_{l+1} .
\end{aligned}
$$

In this equation $j_{e}(=99)$ is the maximum attainable age ${ }^{1} \delta$ the time preference factor, which measures the impatience to consume, $\zeta_{j}$ the conditional (upon being alive at the start of period $j$ ) probability of living through the next year, and $E_{j}$ the expectations operator, i.e. expectations conditional on information available at the start of age $j$. Households derive no utility from leaving bequests. The subjective discount factor consists of two elements. The first element is the already mentioned survival probability which gives a lower weight to per-period utility in more distant years. This survival probability equals the accumulated conditional survival rates $\zeta{ }^{2}$ The second element of the subjective discount factor gives a lower weight to per-period

\footnotetext{
${ }^{1}$ The minimum age of independent decision making is 20 . Children do not supply labour and their material consumption is attributed to their parents. This, in part, accounts for the hump-shaped life-cycle consumption profile used for calibration.

${ }^{2}$ Note, we use as convention $\prod_{l=j}^{j-l} \zeta_{l+1}=1$
} 
utility further in the future due to the impatience of individuals. This impatience element depends on the time preference parameter $\delta$. Per period utility, $u$, is a function of the consumption of commodities, $c_{i}$

$$
u_{i}=\alpha_{i} \frac{c_{i}^{1-\gamma}}{1-\gamma} .
$$

In this equation $1 / \gamma$ is the intertemporal substitution elasticity $(\gamma>1)$ and $\alpha_{i}$ are positive age dependent taste shifters which are used for calibration purposes.

Households have at the start of period $i$ wealth $s_{i}$, they receive a certain income $w_{i}$, consume $c_{i}$ and invest in period $i s_{h i}^{*}$ in assets $h$. Assets have a uncertain return $r_{h i+1}$ which is received at the start of next period $i+1$. So, income from wealth is certain at the start of a period but uncertain for future periods. Households can diversify their mortality risk on the capital market, i.e. they have micro-economic risk but no macro-economic risk. More precisely there is a market that transfers the wealth of the fraction of each cohort that dies in each period $\left(1-\zeta_{i}\right) s_{i}$ to the remaining fraction of that cohort. These assumptions lead to budget equation

$$
\begin{aligned}
\sum_{h} s_{h i}^{*} & =\sum_{h} s_{h i}+w_{i}-c_{i}, \\
s_{h i+1} & =\frac{r_{h i+1}}{\zeta_{i+1}} s_{h i}^{*}, \\
s_{i} & \equiv \sum_{h} s_{h i} .
\end{aligned}
$$

Assume two assets only, a risk free asset $s_{f}$ and a risky asset $s_{s}$ with their respective returns $r_{f}$ and $r_{s}$. The excess return on the risky assets

$$
e_{s i}=r_{s i}-r_{f}
$$

is stochastic. Before the consumption and portfolio decision is made, information about the returns over the investments in previous period become known, i.e. at the start of a period. The stochastic assumptions are

$$
e_{s i}=\mu_{s}+\omega_{s s} \varepsilon_{s i}
$$

$\varepsilon_{s}$ are shocks with expected value 0 and variance 1 . The variance of $e_{s i}$ is

$$
E\left(e_{s i}-\mu_{s}\right)^{2}=\omega_{s s}^{2}
$$

Define $\sigma_{s s}$ as

$$
\sigma_{s s}=E e_{s i}^{2}=\omega_{s s}^{2}+\mu_{s}^{2}
$$

These assumptions of a constant volatility of equity and a constant risk premium imply that mean reversion is absent. 
Households maximize their utility 2.1 given the restrictions 2.3 with respect to consumption $c$ and investments $s_{h i}^{*}$. Using these assumptions appendix B derives household behaviour, which will be discussed now.

\subsection{Household behaviour}

Households allocate total wealth between different periods dependent on relative prices. Total wealth is the sum of financial wealth $s_{j}$, current wages $w_{j}$ and human wealth $h_{j}^{c}$, which is the discounted value of future wage income. The propensity to consume depends on the per period consumption price $p_{c j}$ relative to the price of total wealth $p_{f j}$. Optimal consumption is given by

$$
c_{j}=\left(\frac{p_{c j}}{p_{f j}}\right)^{-\frac{1}{\gamma}}\left(s_{j}+w_{j}+h_{j}^{c}\right)
$$

The total wealth and per period consumption prices are defined as

$$
\begin{aligned}
& p_{f j}=\left[\sum_{i=j}^{j e} p_{c i}^{-\frac{1}{\gamma}}\left(\prod_{l=j}^{i-1}\left(\frac{r_{f}}{\delta}\right)^{\frac{1}{\gamma}} \frac{\zeta_{l+1}}{r_{f}\left[E_{j}\left(1+a e_{s l+1}\right)^{-\gamma}\right]^{-\frac{1}{\gamma}}}\right)\right]^{-\gamma} \\
& p_{c j}=\alpha_{j}^{-1}
\end{aligned}
$$

with $a$ the fraction invested in equities (see B.21). The price of total wealth $p_{f j}$ is the composite price of all future consumption. The weighting factors consists of two elements. The first element relates to substitution over time. A risk-free rate of return above the time preference parameter increases savings, i.e. the price of total wealth, which represents future consumption, decreases. The second term term describes the income effect of returns on investments. A return increase on investments leads to more consumption possibilities not only in the future but in current period, too. The returns on investments consist of three elements, the risk free rate, the survival rate due to our assumption that households participate in a life insurance pool and a certainty equivalence indicator of the excess return on equity investments.

The price of current consumption $p_{c j}$ equals the inverse of the per period consumption preference parameter. A constant fraction of total wealth net of consumption is invested in the risky asset.

$$
s_{s j}^{*}=\operatorname{ar}_{f}\left[s_{j}+w_{j}+h_{j}^{c}-c_{j}\right]
$$

The fraction invested in the risky asset $a$ is implicitly defined by

$$
0=E_{j}\left[\left(1+a e_{s j+1}\right)^{-\gamma} e_{s j+1}\right]
$$

Human wealth $h_{j}^{c}$ is the discounted value of future labour income

$$
h_{j}^{c}=\frac{\zeta_{j+1}}{r_{f}} w_{j+1}+\frac{\zeta_{j+1}}{r_{f}} h_{j+1}^{c}
$$


Section 2.5 presents simulations based on numerical integratior ${ }^{3}$ of equation 2.11 , and of equation $(\mathrm{B} .16)$, a transformation of the excess return certainty equivalence indicator (which appears in the price of total wealth 2.9p. Section 3 presents simulations based on a second-order approximations of both relations.

\section{$2.4 \quad$ Calibration}

The simulations are produced by combining the structure outlined in the previous section with exogenous data and parameters. This section starts with a presentation of these data and parameters. Subsequently a simulation without uncertainty and with uncertainty will be presented.

Consumption and portfolio decisions are made from the age 20 onwards, so consumption of children is attributed to their parents. The values of the parameters are set equal to the parameters in Bovenberg et al. (2007) for convenience. The intertemporal substitution elasticity equals $0.2(\gamma=5)$. The rate of time preference and the risk-free rate take a value of $2 \%$.

Wage income during the working ages, which lay between the ages 20 and 65 , is constant to make the interpretation of the results clear cut. For the same reason, assume a constant consumption profile $\left(\alpha_{j}=1\right)$. The mortality probabilities $\left(1-\zeta_{j}\right)$ are taken from the population forecast of Statistics Netherlands (See: De Jong (2005)).

\section{$2.5 \quad$ Simulations}

The left panel of figure 2.1 presents the results without uncertainty $\left(r_{s i}=r_{f}\right)$. Our assumptions imply a constant consumption. Indeed, the portfolio return equals the time preference parameter. The figure presents the trajectory of financial, human and total wealth. Human wealth depreciates between ages 20 and 65 . Each year human wealth diminishes due to the pay out of the corresponding dividend, the wage stream. Financial wealth increases due to saving for life cycle reasons. At its maximum, financial wealth equals forty four per cent of initial human wealth. Human wealth dominates financial wealth for the most part of the active working life. Figure 2.1 shows that total wealth falls over the life cycle. The nonlinearity in the figure is caused by the multiplicative influence of the rate of return and by the increasing death rates.

The right panel of figure 2.1 presents the results after the introduction of uncertainty. The results based on numerical integration are indicated with (i). The results based on the Taylor

\footnotetext{
3 The integrals are approximated by the Gauss-Hermite quadrature formula with both five and seven nodes. The integration nodes and weights are taken from Judd 1999], Table 7.4.
} 
Figure 2.1 Development of wealth components over the life cycle; without (left) and with uncertainty (right)
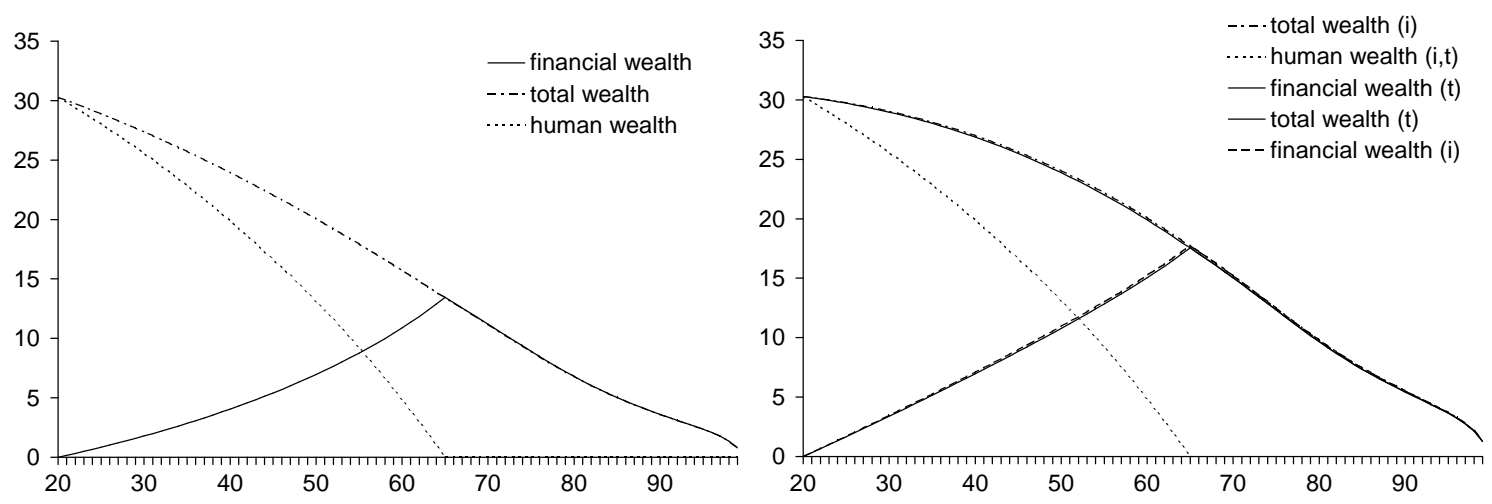

series approximation at $E \varepsilon_{s j}=0$, to be discussed in section 3 are presented with (t). Assume for the variance parameter $\sigma_{s s}=0.04$ and for the excess return $\mu=0.04$ on investments in equity. These assumptions imply a large standard deviation of the excess return $\omega_{s s}=0.2$. The results are presented for the case $\varepsilon_{s i}=0$, i.e. for the case in which everybody behaves as if aggregate uncertainty exists, but without actual shocks. A comparison of the certainty and uncertainty case (using numerical integration) reveals the precautionary saving effect of uncertainty: total wealth decreases more gradually in the uncertainty case relative to the certainty case due to more savings. This precautionary saving effect leads to a $30 \%$ larger maximum financial wealth than in the certainty case at the age of 65 .

\begin{tabular}{lrrrrrrrrrrr}
\hline Table 2.1 & \multicolumn{7}{l}{ Financial indicators household behaviour; the exact solution (numerical integration) } \\
Age & 20 & 21 & 40 & 41 & 64 & 65 & 66 & 80 & 81 & 99 \\
& & & & & & & & & & \\
Wages $w_{j}$ & 1.00 & 1.00 & 1.00 & 1.00 & 1.00 & 0.00 & 0.00 & 0.00 & 0.00 & 0.00 \\
Consumption $c_{j}$ & 0.91 & 0.92 & 1.00 & 1.00 & 1.09 & 1.10 & 1.10 & 1.18 & 1.19 & 1.30 \\
Equity income $r_{s j} s_{s j-1}^{*}$ & 0.00 & 0.37 & 0.33 & 0.33 & 0.22 & 0.22 & 0.21 & 0.12 & 0.11 & 0.01 \\
Bond income $r_{f} s_{f-1}^{*}$ & 0.00 & -0.12 & 0.02 & 0.03 & 0.26 & 0.27 & 0.26 & 0.14 & 0.14 & 0.01 \\
Intra generational redistribution $\zeta_{j} s_{j}$ & 0.00 & 0.00 & 0.01 & 0.01 & 0.12 & 0.13 & 0.14 & 0.41 & 0.43 & 0.39 \\
Financial wealth $s_{j}$ & 0.00 & 0.34 & 7.09 & 7.47 & 17.21 & 17.74 & 17.25 & 9.81 & 9.30 & 1.30 \\
Investments in bonds $s_{f j}^{*}$ & -6.15 & -5.79 & 1.58 & 2.01 & 13.49 & 13.11 & 12.72 & 6.80 & 6.39 & 0.00 \\
Investments in shares $s_{s j}^{*}$ & 6.23 & 6.21 & 5.52 & 5.46 & 3.63 & 3.53 & 3.43 & 1.83 & 1.72 & 0.00 \\
Total wealth $s_{j}+w_{j}+h_{j}^{c}$ & 30.29 & 30.20 & 27.00 & 26.74 & 18.21 & 17.74 & 17.25 & 9.81 & 9.30 & 1.30 \\
Human wealth $w_{j}+h_{j}^{c}$ & 30.29 & 29.86 & 19.90 & 19.27 & 1.00 & 0.00 & 0.00 & 0.00 & 0.00 & 0.00 \\
\hline
\end{tabular}

Table 2.1 details on household behaviour with uncertainty. The table reveals households take debt at the start of their working life to invest in equity with a larger but uncertain return. This portfolio allocation is possible due to the absence of credit restrictions. The average growth rate of consumption of 0.3 per cent per year can be attributed to the introduction of uncertainty. Half 
of this growth rate can be attributed to a larger portfolio return than the time preference parameter. The other half is the precautionary saving effect linked to increased variability.

\section{Taylor approximation of the prudence model}

\subsection{Approximating household behaviour}

We distinguish two different second-order Taylor approximations of the portfolio equation 2.11 and of equation (B.16), a transformation of the excess return certainty equivalence indicator (which appears in the equation for the price of total wealth 2.9) The second-order approximation (see Appendix $\mathrm{B}$ ) of the portfolio equation in the point $e_{s j+1}=0$ leads to an explicit expression for the portfolio share of equity

$$
a=\frac{\mu_{s}}{\gamma \sigma_{s s}}
$$

The investments are proportional to the expected excess return $\mu_{s}$, inversely proportional to $\sigma_{s s}$, which is related to the variance of the excess return and inversely proportional to the risk aversion parameter. The second-order approximation of the portfolio equation in the point $e_{s j+1}=\mu$ leads to another explicit expression for the portfolio share of equity

$$
\widetilde{a}=\left(2 \mu_{s}^{3}-\gamma \mu_{s} \omega_{s s}^{2}+\gamma^{2} \mu_{s} \omega_{s s}^{2}\right)^{-1}\left(-2 \mu_{s}^{2}+\gamma \omega_{s s}^{2}-\omega_{s s} \sqrt{-2 \gamma^{2} \mu_{s}^{2}+\gamma^{2} \omega_{s s}^{2}-2 \gamma \mu_{s}^{2}}\right)
$$

which is approximately equal to

$$
\widehat{a}=\frac{\mu_{s}}{\gamma \omega_{s s}^{2}}
$$

The investments are proportional to the expected excess return $\mu_{s}$, inversely proportional to the variance $\omega_{s s}^{2}$ of the excess return and inversely proportional to the risk aversion parameter. This last expression holds exactly in continuous time models.

\subsection{Simulations}

The parameters and the Taylor series approximation (equation 3.1) imply an equity portfolio share $a=0.2$. The Taylor series approximation overestimates risk aversion relative to numerical integration, which is more accurate. More precisely the equity portfolio share $a=0.208$ is larger in the case of numerical integration. However, the wealth development is nearly the same.

Table 3.1 reveals the accuracy of both the integration and Taylor series expansion method. Increasing the number of nodes from five up to seven in case of numerical integration gives the same portfolio share for equities and the same certainty equivalence indicator of the excess 
Table 3.1 The effect of the number of integration nodes and the approximation point

portfolio share

of equity

Integration

- 5 integration nodes

- 7 integration nodes

Taylor expansion

- in 0

- in $\mu$
0.209

0.208

0.200

0.208 certainty equivalence

indicator

0.997

0.997

return on equity investments $\eta$ (defined in B.16). This indicates that five nodes give already a very good approximation of the exact solution. This is not the case for the Taylor series approximation in the zero excess return point. The approximation in the expected value of the excess return approximates the portfolio share of the exact solution better. The significance of the approximation point is illustrated with table 3.2 and 3.3 . In case of approximation in the expected value $\mu$ (Table 3.3 the differences become smaller relative to the approximation in point zero (Table 3.2). The difference are relative to the central projection of Table 2.1 .

Table 3.2 Absolute differences between the exact solution (numerical integration) and the Taylor series approximation in point $e_{s j}=0$

\begin{tabular}{|c|c|c|c|c|c|c|c|c|c|c|}
\hline Age & 20 & 21 & 40 & 41 & 64 & 65 & 66 & 80 & 81 & 99 \\
\hline Wages & 0.00 & 0.00 & 0.00 & 0.00 & 0.00 & 0.00 & 0.00 & 0.00 & 0.00 & 0.00 \\
\hline Consumption & 0.00 & 0.00 & 0.01 & 0.01 & 0.01 & 0.01 & 0.01 & 0.02 & 0.02 & 0.02 \\
\hline Equity income & 0.00 & 0.01 & 0.01 & 0.01 & 0.01 & 0.01 & 0.01 & 0.01 & 0.01 & 0.00 \\
\hline Bond income & 0.00 & -0.01 & 0.00 & 0.00 & 0.00 & 0.00 & 0.00 & 0.00 & 0.00 & 0.00 \\
\hline Intra generational redistribution & 0.00 & 0.00 & 0.00 & 0.00 & 0.00 & 0.00 & 0.00 & 0.01 & 0.01 & 0.01 \\
\hline Financial wealth & 0.00 & 0.01 & 0.13 & 0.13 & 0.20 & 0.19 & 0.19 & 0.14 & 0.14 & 0.02 \\
\hline Investments in bonds & -0.24 & -0.24 & -0.11 & -0.11 & 0.01 & 0.01 & 0.01 & 0.03 & 0.03 & 0.00 \\
\hline Investments in shares & 0.24 & 0.24 & 0.24 & 0.24 & 0.18 & 0.17 & 0.17 & 0.10 & 0.09 & 0.00 \\
\hline Total wealth & 0.00 & 0.01 & 0.13 & 0.13 & 0.20 & 0.19 & 0.19 & 0.14 & 0.14 & 0.02 \\
\hline Human wealth & 0.00 & 0.00 & 0.00 & 0.00 & 0.00 & 0.00 & 0.00 & 0.00 & 0.00 & 0.00 \\
\hline
\end{tabular}

\section{$4 \quad$ Robustness as explanation for precautionary saving}

\subsection{Introduction}

This section starts in 4.2 with formulating a linear quadratic approximating model of the household problem. Households will not save due to a precautionary saving motive when they 


\begin{tabular}{|c|c|c|c|c|c|c|c|c|c|c|c|}
\hline Table 3.3 & \multicolumn{11}{|c|}{ mation in point $e_{s j}=\mu$} \\
\hline Age & & 20 & 21 & 40 & 41 & 64 & 65 & 66 & 80 & 81 & 99 \\
\hline Wages & & 0.00 & 0.00 & 0.00 & 0.00 & 0.00 & 0.00 & 0.00 & 0.00 & 0.00 & 0.00 \\
\hline Consumpti & & 0.00 & 0.00 & 0.00 & 0.00 & 0.00 & 0.00 & 0.00 & 0.00 & 0.00 & 0.01 \\
\hline Equity inco & & 0.00 & 0.00 & 0.00 & 0.00 & 0.00 & 0.00 & 0.00 & 0.00 & 0.00 & 0.00 \\
\hline Bond incor & & 0.00 & 0.00 & 0.00 & 0.00 & 0.00 & 0.00 & 0.00 & 0.00 & 0.00 & 0.00 \\
\hline Intra gener & ational redistribution & 0.00 & 0.00 & 0.00 & 0.00 & 0.00 & 0.00 & 0.00 & 0.00 & 0.00 & 0.00 \\
\hline Financial n & ealth & 0.00 & 0.00 & 0.03 & 0.03 & 0.05 & 0.05 & 0.05 & 0.04 & 0.03 & 0.01 \\
\hline Investment & s in bonds & 0.01 & 0.01 & 0.04 & 0.04 & 0.04 & 0.04 & 0.04 & 0.03 & 0.03 & 0.00 \\
\hline Investment & $s$ in shares & -0.01 & -0.01 & 0.00 & 0.00 & 0.00 & 0.00 & 0.00 & 0.00 & 0.00 & 0.00 \\
\hline Total wealt & & 0.00 & 0.00 & 0.03 & 0.03 & 0.05 & 0.05 & 0.05 & 0.04 & 0.03 & 0.01 \\
\hline Human we & alth & 0.00 & 0.00 & 0.00 & 0.00 & 0.00 & 0.00 & 0.00 & 0.00 & 0.00 & 0.00 \\
\hline
\end{tabular}

use quadratic utility. However when they realize their incomplete knowledge about the real world, i.e. they have an approximating model of the real world, precautionary saving will occur because they will plan using a worst case scenario. Indeed, this will be the case when households use robust control to determine their behaviour. Subsection 4.3 digresses on robust control. Household behaviour comes into discussion in 4.4 Subsection 4.5 presents the simulation results.

\subsection{A linear quadratic model of the household decision}

The household problem formulated in section 2.2 can be written as

$$
V_{j}=\max E_{j}\left[\frac{\alpha_{j}}{1-\gamma} c_{j}^{1-\gamma}+\delta^{-1} \zeta_{j+1} V_{j+1}\right]
$$

with

$$
c_{j}=\frac{r_{f}}{\zeta_{j}} s_{j-1}^{*}+\frac{e_{s j}}{\zeta_{j}} a r_{f}\left[s_{j-1}^{*}+h_{j-1}^{c}\right]+w_{j}-s_{j}^{*}
$$

in which $s_{j}^{*}\left(=\sum_{h} s_{h j}^{*}\right)$ the total portfolio invested in $j$ and $a$, as before, the fraction of wealth invested in the risky asset. Assume, the fraction invested in the risky asset is known for convenience. More specifically, the fraction is determined by equation 3.1 . So, the robust model assumes in fact only one asset with a stochastic return. The objective function is maximized given the linear restriction

$$
e_{s j+1}=\mu_{s}+\omega_{s s} \varepsilon_{s j}
$$

This equation corresponds with equation 2.5. Households do not derive utility from bequests, this implies that they don't invest at the end of their life $\left(s_{j e}^{*}=0\right)$, i.e.in the end year $j=j e$. This 
implies for the value function

$$
V_{j}\left(s_{j e-1}\right)=\alpha_{j e} \frac{c_{j e}^{1-\gamma}}{1-\gamma}
$$

The problem is thus written as the maximization of a nonlinear objective function subject to one linear constraint. The objective function (i.e. equation 4.1 after substitution of 4.2 ) can be approximated around the non-stochastic version of the nonlinear model (with $a=0.2$ and $e_{s j}=0 \sqrt{4}$ This leads to a linear quadratic Gaussian control problem 5

$$
\begin{aligned}
\max _{\mathbf{u}_{j}} V_{j}\left(\mathbf{x}_{j}\right) & =-\mathbf{x}_{j}^{\prime} \mathbf{R}_{j} \mathbf{x}_{j}-\mathbf{u}_{j}^{\prime} \mathbf{Q}_{j} \mathbf{u}_{j}+\delta^{-1} E_{j} \zeta_{j+1} V\left(\mathbf{x}_{j+1}\right) \\
\mathbf{x}_{j+1} & =\mathbf{A}_{j} \mathbf{x}_{j}+\mathbf{B} \mathbf{u}_{j}+\mathbf{C} \varepsilon_{j+1} \\
E \varepsilon \varepsilon^{\prime} & =\mathbf{I}
\end{aligned}
$$

and

$$
V_{j e}\left(\mathbf{x}_{j e}\right)=-\mathbf{x}_{j e}^{\prime} \mathbf{R}_{j e} \mathbf{x}_{j e}
$$

with $\mathbf{x}$ the vector of state variables, $\mathbf{u}$ the vector of control variables. The elements of the state vector $\mathbf{x}$ are financial wealth $s^{*}$, the excess return $e_{s}$ and constant term 1 . The vector of control variables $\mathbf{u}$ has only one element: a transformation ${ }^{6}$ of $s^{*}$ the investments in financial wealth in current period. Note the covariance matrix $E_{j}\left(\mathbf{x}_{j+1}-E_{j} \mathbf{x}_{j+1}\right)\left(\mathbf{x}_{j+1}-E_{j} \mathbf{x}_{j+1}\right)^{\prime}=\mathbf{C C}^{\prime}$.

This procedure is known as the Kydland and Prescot method (Benigno and Woodford (2006) discuss this method and give extensions). This procedure results in a correct local linear approximation to the optimal policy belonging to the original problem. However, precautionary saving is based on the third derivatives of the utility function. So using Linear Quadratic Gaussian control (LQG) leads to misspecified dynamics due to misspecified utility. There is another reason to consider this model as an approximating model for household behaviour. To take into account the approximating nature of the linear quadratic model robust control can be used. The knowledge of households that they have only an approximating model of the world, i.e. of the excess return process $e_{s j}$, leads to precautionary saving.

\footnotetext{
${ }^{4}$ We linearize in $a=0.2$ to get non zero derivatives of the excess return.

${ }^{5}$ Appendix $\mathrm{C}$ shows that the linear terms can be included in the quadratic one by defining an additional help-vector.

${ }^{6}$ Appendix C.1 gives details.
} 
Robust contro $]^{7}$ was developed to deal with the idea that agents have incomplete knowledge about the world. More specifically, robust control assumes that the decision maker thinks that his model (equation 4.5) approximates the true data generating process, which he cannot specify. The error terms in 4.5 can represent only a very limited class of approximation errors and in particular cannot account for misspecified dynamics. To represent dynamic misspecification, the decision maker surrounds 4.5 with a set of alternative models of the form

$$
\begin{aligned}
\mathbf{x}_{j+1} & =\mathbf{A}_{j} \mathbf{x}_{j}+\mathbf{B} \mathbf{u}_{j}+\mathbf{C}\left(\varepsilon_{j+1}+\mathbf{a}_{j+1}\right) \\
\mathbf{a}_{j+1} & =b_{j}\left(\mathbf{x}_{j}, \mathbf{x}_{j-1}, \ldots\right) \\
E \varepsilon \varepsilon^{\prime} & =\mathbf{I}
\end{aligned}
$$

The decision maker believes that the data are generated by a model of the form 4.8 with unknown process $\mathbf{a}_{j}$. In case $\varepsilon$ in 4.8 has a distribution $N(0, \mathbf{I})$ then model 4.5 must be misspecified because its error term will be distributed $N\left(\mathbf{a}_{j+1}, \mathbf{I}\right)$ rather than $N(0, \mathbf{I})$. However, agents know that model 4.5 is a good approximation if the actual model is located in the surround 4.8 . So, robust control assumes uncertainty about the autocorrelation $\left(\mathbf{a}_{j}\right.$ depends on the lagged state vector $\mathbf{x}_{j}$ ) and abstracts from misspecification of higher moment of the $\varepsilon_{j+1}$ distribution. Moreover there is no uncertainty about the modelparameters (the variance covariance structure, determined by $C$, is hold constant, too). Hansen and Sargent (2007) show this specification is not as restrictive as it might at first seem. To express the idea that 4.5 is a good approximation we constrain the approximation errors by

$$
E_{j} \sum_{i} \delta^{-1} \zeta_{i+1} \mathbf{a}_{i+1} \mathbf{a}_{i+1}<\chi_{j}
$$

where $E$ denotes the mathematical expectation and $\chi_{j}$ a parameter to be calibrated. This introduced parameter $\chi_{j}$ is without a counterpart in the prudence approach (This parameter is related to the separate risk aversion parameter in the Epstein-Zin utility function as Tallarini (2000) shows). The restriction 4.9 is known as the entropy constraint. The decision maker want good decisions over a set of models 4.8 satisfying entropy constraint 4.9 because he distrusts model 4.5. Such decisions are said to be robust to model misspecification. So, robust control states that households maximize the (forward solution of) objective function 4.4 subject to this actual law of motion 4.8 and the entropy constraint 4.9 given an estimated worst case scenario, i.e. households maximize (the forward solution of) 4.4 with respect to $u$ after minimizing (the forward solution of) 4.4 over $a$ given the constraints 4.8 and 4.9 , which is under some regularity

\footnotetext{
7 This section is based on Hansen and Sargent 2007.
} 
conditions equal to[

$$
\begin{aligned}
V_{j}\left(\mathbf{x}_{j}\right) & =\max _{\mathbf{u}_{j}} \operatorname{anin}_{j+1} E_{j}\left[-\mathbf{x}_{j}^{\prime} \mathbf{R}_{j} \mathbf{x}_{j}-\mathbf{u}_{j}^{\prime} \mathbf{Q}_{j} \mathbf{u}_{j}+\right. \\
& \left.+\theta \delta^{-1} \zeta_{j+1} \mathbf{a}_{j+1}^{\prime} \mathbf{a}_{j+1}+\delta^{-1} \zeta_{j+1} V_{j+1}\left(\mathbf{x}_{j+1}\right)\right]
\end{aligned}
$$

given the distorted model 4.8 The parameter $\theta$ is related to the entropy measure $\chi$. It restrains the minimizing choice of $a_{j}$ and determines the degree of risk aversion.

\subsection{Household behaviour}

The robust decision rule is a linear function of the state of the economy $(j<j e)$

$$
\mathbf{u}_{j}=-\mathbf{F}_{j} \mathbf{x}_{j}
$$

just as in the standard LQG problem. The decision maker will combine his approximating model 4.5 with this robust decision rule. These simple relations lead to a recursive closed form solution for the state of the economy

$$
\mathbf{x}_{j+1}=\left(\mathbf{A}_{j}-\mathbf{B F} \mathbf{F}_{j}\right) \mathbf{x}_{j}+\mathbf{C} \varepsilon_{j+1}
$$

which makes stochastic simulation easy to handle in case the decision maker's fear of model misspecification is unfounded, i.e. $\varepsilon_{j+1} \sim N(0, \mathbf{I})$. Without shocks, equation 4.13 determines an investment profile over the life cycle. Shocks will cause parallel movements with this investment profile. The Consumption and portfolio allocation can be recursively determined after the investments are fixed.

\subsection{Simulations using a robust linear regulator}

The left panel of Figure 4.1 compares the simulation results using the robust linear regulator (indicated with $r$ ) with the results based on the Taylor series approximation in the zero excess return point of the prudence model (indicated with a $t$ ). The entropy related parameter $(\theta=7)$ is calibrated such that the consumption at the age of 20 equals the consumption in the prudence model (see the right panel). The right panel presents consumption also for $\theta=\infty$ (indicated with z) which is equal to the standard Linear Quadratic Gaussian (LQG) control solution. Comparing consumption for the two different values of $\theta$ reveals the precautionary saving effect at the start of the working ages. Buffers are built up early in life to meet uncertainty after which consumption becomes a little bit larger later on. The difference between the robust control and prudence model has to be attributed to the difference in the intertemporal substitution elasticity. Linearization in the expected excess return will bring them more in line. 
Figure 4.1 Development of wealth components over the life cycle (left) and consumption over the life cycle (right)
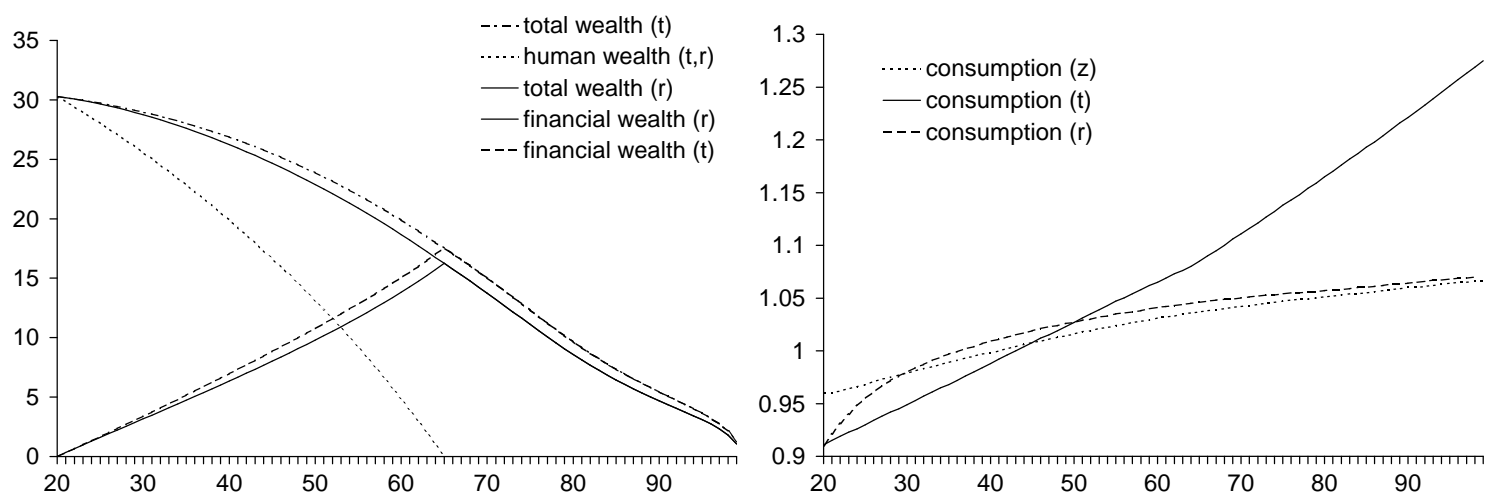

Table 4.1 Financial indicators household behaviour; the robust solution

\begin{tabular}{lrrrrrrrrrr} 
Age & 20 & 21 & 40 & 41 & 64 & 65 & 66 & 80 & 81 & 99 \\
Wages $w_{j}$ & & & & & & & & & & \\
Consumption $c_{j}$ & 1.00 & 1.00 & 1.00 & 1.00 & 1.00 & 0.00 & 0.00 & 0.00 & 0.00 & 0.00 \\
Equity income $r_{s j} s_{s j-1}^{*}$ & 0.91 & 0.92 & 1.01 & 1.01 & 1.05 & 1.05 & 1.05 & 1.06 & 1.06 & 1.07 \\
Bond income $r_{s j} s_{f-1}^{*}$ & 0.00 & 0.36 & 0.31 & 0.31 & 0.20 & 0.19 & 0.19 & 0.10 & 0.09 & 0.01 \\
Intra generational redistribution $\zeta_{j} s_{j}$ & 0.00 & 0.00 & 0.00 & 0.01 & 0.11 & 0.12 & 0.13 & 0.36 & 0.38 & 0.32 \\
Financial wealth $s_{j}$ & 0.00 & 0.33 & 6.34 & 6.67 & 15.74 & 16.25 & 15.76 & 8.59 & 8.12 & 1.07 \\
Investments in bonds $s_{f j}^{*}$ & -5.90 & -5.56 & 1.18 & 1.57 & 12.49 & 12.11 & 11.71 & 6.00 & 5.62 & 0.00 \\
Investments in shares $s_{s j}^{*}$ & 5.99 & 5.97 & 5.15 & 5.09 & 3.20 & 3.10 & 3.00 & 1.54 & 1.44 & 0.00 \\
Total wealth $s_{j}+w_{j}+h_{j}^{c}$ & 30.29 & 30.19 & 26.24 & 25.95 & 16.74 & 16.25 & 15.76 & 8.59 & 8.12 & 1.07 \\
Human wealth $w_{j}+h_{j}^{c}$ & 30.29 & 29.86 & 19.90 & 19.27 & 1.00 & 0.00 & 0.00 & 0.00 & 0.00 & 0.00 \\
\hline
\end{tabular}

Table 4.1 presents the development of some financial indicators of household behaviour in case of robust control. They illustrate the main graphical results. The difference with the prudence model are given in table 4.2

The robust control outcomes can approximate the prudence outcome by changing the time preference parameter $\delta$ and the entropy parameter $\theta$. With $\theta=1.9$ and $\delta=.075$ we get the results Figure 4.2 and table 4.3 However, these parameter adjustments have huge impact on the dynamic characteristics of the model. This is illustrated in figure 4.3 At the start of the working life a negative equity premium shock $\left(\varepsilon_{s i}=-1\right)$ takes place. The prudence model (Taylor series approximation in $e_{s j+1}=0$ ) gives a rather constant adjustment. The robust results without adjustment of the time preference parameter (robust 1) gives a large precautionary saving effect in the first years. After increasing the time preference parameter the consumption adjustment are large at the end of the life cycle. Figure 4.4 presents both the central projection 
Table 4.2 Absolute differences between the robust solution and the Taylor series approximation in point $e_{s j}=0$

\begin{tabular}{|c|c|c|c|c|c|c|c|c|c|c|}
\hline Age & 20 & 21 & 40 & 41 & 64 & 65 & 66 & 80 & 81 & 99 \\
\hline Wages & 0.00 & 0.00 & 0.00 & 0.00 & 0.00 & 0.00 & 0.00 & 0.00 & 0.00 & 0.00 \\
\hline Consumption & 0.00 & -0.01 & -0.02 & -0.02 & 0.04 & 0.04 & 0.04 & 0.11 & 0.11 & 0.21 \\
\hline Equity income & 0.00 & 0.00 & 0.01 & 0.01 & 0.02 & 0.02 & 0.02 & 0.01 & 0.01 & 0.00 \\
\hline Bond income & 0.00 & 0.00 & 0.01 & 0.01 & 0.02 & 0.02 & 0.02 & 0.02 & 0.02 & 0.00 \\
\hline Intra generational redistribution & 0.00 & 0.00 & 0.00 & 0.00 & 0.01 & 0.01 & 0.01 & 0.05 & 0.05 & 0.06 \\
\hline Financial wealth & 0.00 & 0.00 & 0.63 & 0.66 & 1.28 & 1.29 & 1.30 & 1.08 & 1.05 & 0.21 \\
\hline Investments in bonds & 0.00 & 0.00 & 0.51 & 0.54 & 0.99 & 1.00 & 1.00 & 0.77 & 0.74 & 0.00 \\
\hline Investments in shares & 0.00 & 0.00 & 0.13 & 0.14 & 0.25 & 0.26 & 0.26 & 0.20 & 0.19 & 0.00 \\
\hline Total wealth & 0.00 & 0.00 & 0.63 & 0.66 & 1.28 & 1.29 & 1.30 & 1.08 & 1.05 & 0.21 \\
\hline Human wealth & 0.00 & 0.00 & 0.00 & 0.00 & 0.00 & 0.00 & 0.00 & 0.00 & 0.00 & 0.00 \\
\hline
\end{tabular}

indicated with $c$ and the alternative path indicated with $a$ with the equity return shock for both consumption and wealth. The results for the robust model in this figure are the results after the parameter adjustments.

Figure 4.2 Development of wealth components over the life cycle (left) and consumption over the life cycle (right)
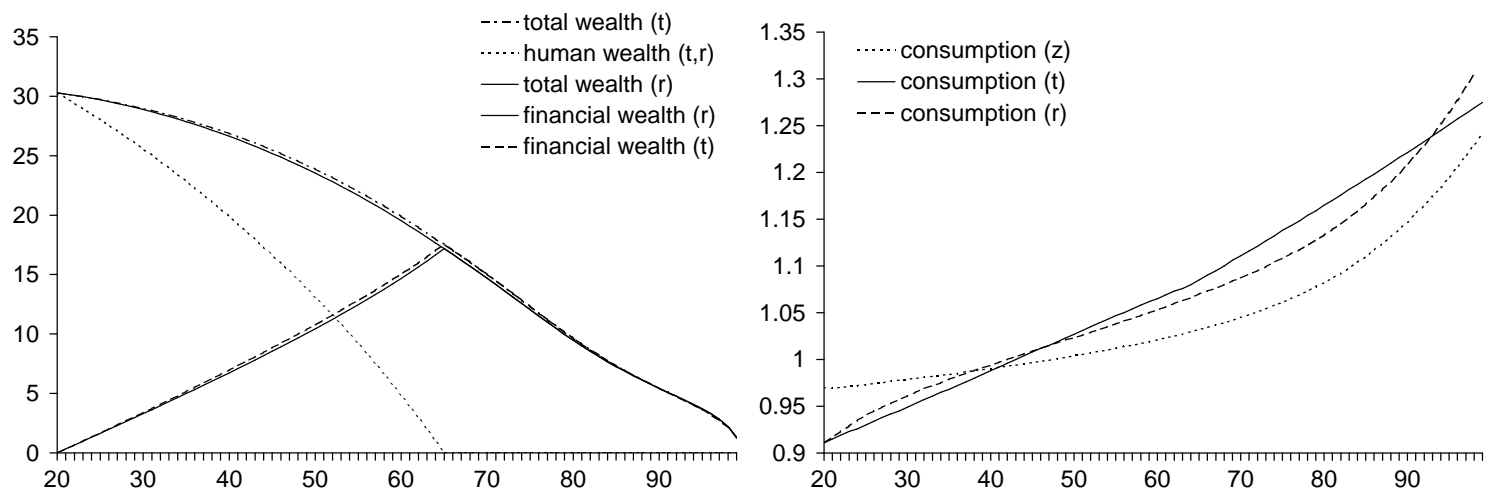


\begin{tabular}{lrrrrrrrrrrr}
\hline \multicolumn{7}{l}{ Table 4.3 } & \multicolumn{7}{c}{ Absolute differences between the robust solution and the Taylor series approximation in point $e_{s j}=0$} \\
Age & 20 & 21 & 40 & 41 & 64 & 65 & 66 & 80 & 81 & 99 \\
& & & & & & & & & & \\
Wages & 0.00 & 0.00 & 0.00 & 0.00 & 0.00 & 0.00 & 0.00 & 0.00 & 0.00 & 0.00 \\
Consumption & 0.00 & 0.00 & -0.01 & -0.01 & 0.01 & 0.01 & 0.02 & 0.03 & 0.03 & -0.05 \\
Equity income & 0.00 & 0.00 & 0.00 & 0.00 & 0.00 & 0.00 & 0.00 & 0.00 & 0.00 & 0.00 \\
Bond income & 0.00 & 0.00 & 0.00 & 0.00 & 0.01 & 0.01 & 0.01 & 0.00 & 0.00 & 0.00 \\
Intra generational redistribution & 0.00 & 0.00 & 0.00 & 0.00 & 0.00 & 0.00 & 0.00 & 0.01 & 0.01 & -0.01 \\
Financial wealth & 0.00 & 0.00 & 0.23 & 0.25 & 0.37 & 0.37 & 0.37 & 0.19 & 0.17 & -0.05 \\
Investments in bonds & 0.00 & 0.00 & 0.19 & 0.20 & 0.28 & 0.28 & 0.28 & 0.12 & 0.11 & 0.00 \\
Investments in shares & 0.00 & 0.00 & 0.05 & 0.05 & 0.07 & 0.07 & 0.07 & 0.03 & 0.03 & 0.00 \\
Total wealth & 0.00 & 0.00 & 0.23 & 0.25 & 0.37 & 0.37 & 0.37 & 0.19 & 0.17 & -0.05 \\
Human wealth & 0.00 & 0.00 & 0.00 & 0.00 & 0.00 & 0.00 & 0.00 & 0.00 & 0.00 & 0.00 \\
\hline
\end{tabular}

\section{Summary}

This paper compares two models of household behaviour that can explain precautionary saving, i.e. saving associated with the variability of exogenous random shocks. The prudence model assumes convexity of the marginal utility function. The distrust model is based on robust decision making given incomplete knowledge about the real world. The prudence model leads to first-order conditions for optimal behaviour in which expectations play a role. Numerical integration is compared with Taylor series expansion to get rid of this expectation term. We find

Figure 4.3 Absolute consumption changes over the life cycle after an equity premium shock

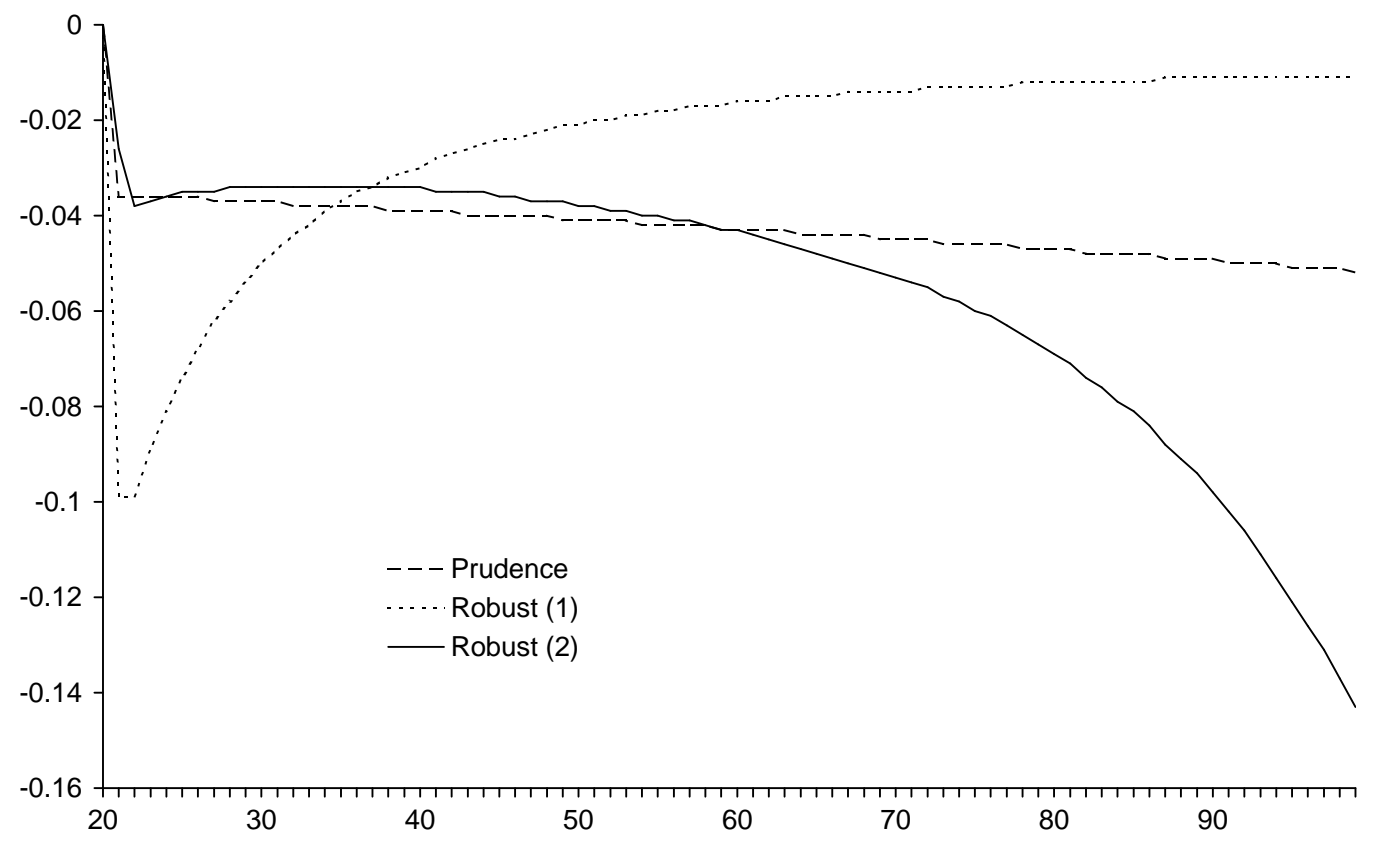


Figure 4.4 Development of consumption and financial wealth over the life cycle; prudence (left) robust (right)

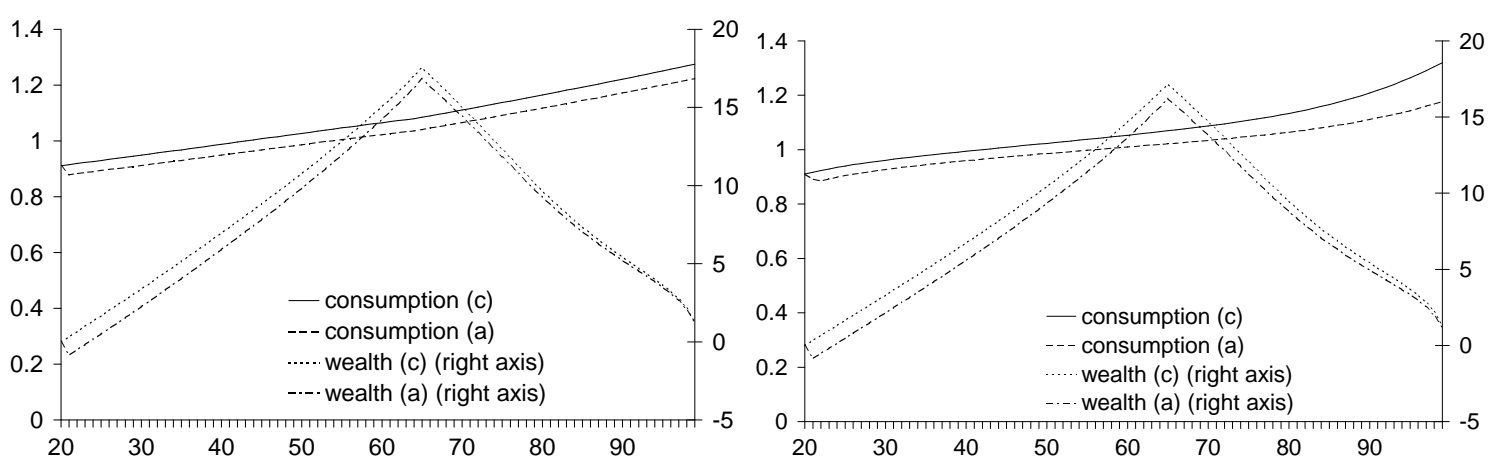

that Taylor series approximation can lead to the same results as numerical integration dependent on the point of linearization. Robust control leads to linear decision rules which need no further approximations. The robust model is found to approximate the outcomes of the prudence model reasonably well after increasing the time preference parameter and decreasing the entropy related parameter.

Two caveats are in order. The first is that the comparison of different approaches in this paper adopts a stylized model. We have no guarantee that the results for this stylized model can be exported to models that are more detailed. The second caveat is that our list of approaches is incomplete. For example, we did not pay attention to the linearization approach that is introduced by Campbell and Viceira (see for instance Viceira (2001)), although we feel that their approach is very close to our Taylor series approximation. Their portfolio allocation equation is exactly ours although their derivation is different. Their approach seems more appropriate in a representative agents framework while the approach adopted here is more natural in an overlapping generation context. We also did not pay attention to perturbation methods as discussed by Judd (1999). Perturbation is a numerical method to obtain first and higher-order Taylor expansions. Due to the numerical approach the applicability is larger. For instance, Taylor series expansions can be obtained of rational expectations policy functions around the steady state. 


\section{Appendix A Symbols main text}

\section{Matrices}
A coefficient matrix model
B coefficient matrix model
C coefficient matrix model, determining variance covariances of the state variables
F policy function matrix
Q utility weights control variables
R utility weights state variables

Vectors
a distortion vector
$\mathbf{x}$ state variables
u control variables

\section{Variables}

$a_{1}$ fraction invested in equity

c consumption

$d_{s}$ subjective discount factor

$e_{s}$ excess return equities

$h^{c}$ human capital

$r_{h}$ rate of return asset $h \in\{f, s\}$

$p_{c}$ consumption price

$p_{f}$ price total wealth

$s$ total amount invested in assets; begin of the period, before the new portfolio decision

$s^{*}$ total amount invested in assets; begin of the period, after the new portfolio decision

$s_{h}$ amount invested in asset $h \in\{f, s\}$; begin of the period, before the new portfolio decision

$s_{h}^{*}$ amount invested in asset $h \in\{f, s\}$; begin of the period, after the new portfolio decision

$U$ expected utility

$u$ instantaneous utility

$V$ value function

$w$ wages

coefficients and shock term

$\alpha$ taste shifter 
$\gamma$ inverse intertemporal substitution coefficient

$\delta$ time preference

$\varepsilon_{s}$ stochastic shock term equity

$\zeta$ survival rate

$\theta$ entropy related coefficient

$\mu$ expected value excess return

$\sigma_{s s}$ variance indicator equity

$\omega_{s s}$ standard deviation excess return equity

$\chi$ entropy measure

Operators

$E$ expectation operator 


\section{Appendix B Derivations section 2 and 3}

Making use of the value function $V_{j}\left(s_{j}\right)=\max U_{j}$ the decision problem 2.1 can be written as a sequence of one period decisions

$$
V_{j}\left(s_{j}\right)=\max \left(u_{j}+\delta^{-1} \zeta_{j+1} E_{j} V_{j+1}\left(s_{j+1}\right)\right)
$$

Note

$$
s_{j+1}=\frac{r_{f}}{\zeta_{j+1}}\left(s_{j}+w_{j}-c_{j}\right)+\frac{e_{j+1}}{\zeta_{j+1}} s_{s j}^{*}
$$

The lagrangian of the problem is

$$
L=u_{j}+\delta^{-1} \zeta_{j+1} E_{j}\left(V_{j+1}\left(\sum_{h} \frac{r_{h j+1}}{\zeta_{j+1}} s_{h j}^{*}\right)+\lambda_{j}\left[\sum_{h}\left(s_{h j}^{*}-s_{h j}\right)-w_{j}+c_{j}\right]\right)
$$

First order conditions are

$$
\begin{aligned}
& \text { ı. } \frac{\partial L}{\partial c_{j}}=u_{j}^{\prime}+\delta^{-1} \zeta_{j+1} E_{j} \lambda_{j}=0 \\
& \text { ıı. } \frac{\partial L}{\partial s_{h j}^{*}}=\delta^{-1} \zeta_{j+1}\left(E_{j}\left(\frac{\partial V_{j+1}}{\partial s_{j+1}} \frac{r_{h j+1}}{\zeta_{j+1}}\right)+E_{j} \lambda_{j}\right)=0
\end{aligned}
$$

From the Belmann equation follows

$$
\text { ıı. } \frac{\partial V_{j}}{\partial s_{h j}}=\delta^{-1} \zeta_{j+1} E_{j} \frac{\partial V_{j+1}}{\partial s_{j+1}} \frac{r_{h j+1}}{\zeta_{j+1}}
$$

Subsitution of $\imath \iota$ into $\imath \iota \imath$ gives the envelop theorem

$$
\frac{\partial V_{j}}{\partial s_{h j}}=-\delta^{-1} \zeta_{j+1} E_{j} \lambda_{j}=u_{j}^{\prime}
$$

Substitution of this relation into equation $1 \imath \iota$ gives the two Euler equations

$$
\imath v . u_{j}^{\prime}=E_{j} u_{j+1}^{\prime} \frac{r_{h j+1}}{\delta}
$$

Subtraction of the two Euler equations (for $h \in\{s, f\}$ ) and using the excess return definition gives

$$
0=E_{j} u_{j+1}^{\prime} e_{s j+1}
$$

Substitution of the marginal utility into $l$ gives

$$
\text { t. } \alpha_{j} c_{j}^{-\gamma}+\delta^{-1} \zeta_{j+1} E_{j} \lambda_{j}=0
$$


Assume the value function has the form

$$
V_{j}\left(s_{j}\right)=k_{j}^{\gamma} \frac{\left(s_{j}+w_{j}+h_{j}^{c}\right)^{1-\gamma}}{1-\gamma}+l_{j}
$$

for some constants $h_{j}^{c} \ldots h_{j e}^{c}, k_{j} \ldots k_{j e}, l_{j} \ldots l_{j e}$ to be specified. We assume non stochastic $h^{c}, k$ and $l$ this assumption has to be checked at the end of the derivation. The derivative of the value function becomes

$$
\frac{\partial V_{j}}{\partial s_{h j}}=k_{j}^{\gamma}\left(s_{j}+w_{j}+h_{j}^{c}\right)^{-\gamma}
$$

Substitute this result and envelop theorem B.7 into B.9 subsitute budget equation B.2, and devide both sides by the non stochastic part, which leads $\mathrm{tg}^{8}$

$$
0=E_{j}\left(1+a e_{s j+1}\right)^{-\gamma} e_{s j+1}
$$

with

$$
a=\frac{s_{s j}^{*}}{r_{f}\left(s_{j}+w_{j}-c_{j}\right)+\zeta_{j+1} w_{j+1}+\zeta_{j+1} h_{j+1}^{c}}
$$

So, we derived an implicit equation for the portfolio share invested in the risky asset. To get an expression for $h_{j}^{c}$ we need to solve the consumption decision first. Subsitute first order condition B.5 into first order condition B.4 substitute subsequently the value function B.12 and utility function derivatives for the case $h=f$. This leads after subsitution of budget equation B.2 to

$$
c_{j}=\frac{r_{f}}{k_{j+1} \eta_{j+1} \zeta_{j+1}+r_{f}}\left(s_{j}+w_{j}+\frac{\zeta_{j+1}}{r_{f}} w_{j+1}+\frac{\zeta_{j+1}}{r_{f}} h_{j+1}^{c}\right)
$$

with

$$
\eta_{j+1}=\left(\frac{r_{f}}{\delta \alpha_{j}} E_{j}\left(1+a e_{s j+1}\right)^{-\gamma}\right)^{\frac{1}{\gamma}}
$$

Next we have to derive formulas for $h^{c}$ and $k$. Use the envelop equation B.7, subsitute the derivative of the value function B.12 the derivative of the instantaneous utility function and consumption equation B.15

$$
k_{j}^{-1}\left(s_{j}+w_{j}+h_{j}^{c}\right)=\alpha_{j}^{-\frac{1}{\gamma}} \frac{r_{f}}{k_{j+1} \eta_{j+1} \zeta_{j+1}+r_{f}}\left(s_{j}+w_{j}+\frac{\zeta_{j+1}}{r_{f}} w_{j+1}+\frac{\zeta_{j+1}}{r_{f}} h_{j+1}^{c}\right)
$$

which leads to

$$
k_{j}=\alpha_{j}^{\frac{1}{\gamma}} \frac{k_{j+1} \eta_{j+1} \zeta_{j+1}+r_{f}}{r_{f}}
$$

${ }^{8}$ Note, we assume $k_{j}^{\gamma} \neq 0$ and $\zeta_{j}^{\gamma} \neq 0$ 


$$
h_{j}^{c}=\frac{\zeta_{j+1}}{r_{f}} w_{j+1}+\frac{\zeta_{j+1}}{r_{f}} h_{j+1}^{c}
$$

which is consistent with our assumption that $k_{j}$ and $h_{j}^{c}$ are non-stochastic. Solving the equation forewards leads to

$$
k_{j}=\sum_{i=j}^{j e} \alpha_{i}^{\frac{1}{\gamma}}\left(\prod_{l=j}^{i-1} \alpha_{l}^{\frac{1}{\gamma}} \eta_{l+1} \zeta_{l+1} r_{f}^{-1}\right) \equiv p_{f j}^{-\frac{1}{\gamma}}
$$

which entails for the propensity to consume

$$
k_{j+1} \eta_{j+1} \zeta_{j+1} r_{f}^{-1}+1=p_{f j}^{-\frac{1}{\gamma}} \alpha_{j}^{-\frac{1}{\gamma}} \equiv p_{f j}^{-\frac{1}{\gamma}} p_{c j}^{\frac{1}{\gamma}}
$$

Subsitution into B.15 leads to consumption equation 2.8

We have equations of the form $f(y)=(1+a y)^{-\gamma} y$ and $g(y)=(1+a y)^{-\gamma}$. These equations will be subsequently approximated in $y_{0}=0$ and in $y_{0}=\mu$. The most simple expressions are obtained in $y_{0}=0$. The second order Taylor series approximations become

$$
f(y) \approx y-\gamma a y^{2}
$$

$$
g(y) \approx 1-\gamma a y+\frac{1}{2} \gamma(\gamma+1) a^{2} y^{2}
$$

Subsitute the approximation for $f$ into portfolio equation $\mathrm{B.13}$ to obtain

$$
0 \approx \mu_{s}-\gamma a \sigma_{s s}
$$

which leads to portfolio equation 3.1 Subsitute the aproximation for $g$ into B.16 to obtain

$$
\eta_{j+1} \approx\left(\frac{r_{f}}{\delta \alpha_{j}}\left(1-\gamma a \mu_{s}+\frac{1}{2} \gamma(\gamma+1) a^{2} \sigma_{s s}\right)\right)^{\frac{1}{\gamma}}
$$

Approximation of $f$ in $y_{0}=\mu_{s}$ leads to

$$
\begin{aligned}
f(y) & \approx\left(1+a \mu_{s}\right)^{-\gamma} \mu_{s} \\
& +\left[\left(1+a \mu_{s}\right)^{-\gamma}-\gamma a\left(1+a \mu_{s}\right)^{-\gamma-1} \mu_{s}\right]\left(y-\mu_{s}\right) \\
& +\frac{1}{2}\left[-2 \gamma a\left(1+a \mu_{s}\right)^{-\gamma-1}+\gamma(\gamma+1) a^{2}\left(1+a \mu_{s}\right)^{-\gamma-2} \mu_{s}\right]\left(y-\mu_{s}\right)^{2}
\end{aligned}
$$

Approximate $g(y)=(1+a y)^{-\gamma}$ in $\mu_{s}$ using a second order Taylor series

$$
g(y) \approx\left(1+a \mu_{s}\right)^{-\gamma}-\gamma a\left(1+a \mu_{s}\right)^{-\gamma-1}\left(y-\mu_{s}\right)+\frac{1}{2} \gamma(\gamma+1) a^{2}\left(1+a \mu_{s}\right)^{-\gamma-2}\left(y-\mu_{s}\right)^{2}
$$


Substitute approximation B.26 into B.13 to obtain

$$
0 \approx \frac{1}{2} a^{2} \gamma^{2} \mu_{s} \omega_{s s}^{2}-\frac{1}{2} a^{2} \gamma \mu_{s} \omega_{s s}^{2}+a^{2} \mu_{s}^{3}-a \gamma \omega_{s s}^{2}+2 a \mu_{s}^{2}+\mu_{s}
$$

which leads to portfolio equation 9

$$
\tilde{a}=\frac{1}{2 \mu_{s}^{3}-\gamma \mu_{s} \omega_{s s}^{2}+\gamma^{2} \mu_{s} \omega_{s s}^{2}}\left(-2 \mu_{s}^{2}+\gamma \omega_{s s}^{2}-\omega_{s s} \sqrt{-2 \gamma^{2} \mu_{s}^{2}+\gamma^{2} \omega_{s s}^{2}-2 \gamma \mu_{s}^{2}}\right)
$$

Substitute the approximation B.27 for $g$ into B.16 to obtain

$$
\widetilde{\eta}_{j+1} \approx\left(\frac{r_{f}}{\delta \alpha_{j}}\left(\left(1+a \mu_{s}\right)^{-\gamma}+\frac{1}{2} \gamma(\gamma+1) a^{2}(1+a \mu)^{-\gamma-2} \omega_{s s}^{2}\right)\right)^{\frac{1}{\gamma}}
$$

Another approximation is obtained after substitution of B.26 into B.13, i.e.

$$
\begin{aligned}
0 & =\left(1+a \mu_{s}\right)^{-\gamma} \mu_{s} \\
& +\frac{1}{2}\left[-2 \gamma a\left(1+a \mu_{s}\right)^{-\gamma-1}+\gamma(\gamma+1) a^{2}\left(1+a \mu_{s}\right)^{-\gamma-2} \mu_{s}\right] \omega_{s s}^{2}
\end{aligned}
$$

Make use of $\left(1+a \mu_{s}\right)^{-1}-\frac{1}{2}(\gamma+1) a\left(1+a \mu_{s}\right)^{-2} \mu_{s} \approx 1$ to get

$$
\widehat{a} \approx \frac{\mu_{s}}{\gamma \omega_{s s}^{2}}
$$

Lastly note that no investments occur at the maximum attainable age $s_{s j e}^{*}=0$, moreover human wealth is zero $h_{j e}^{c}=0$, and

$$
k_{j e}=\alpha_{j e}^{\frac{1}{\gamma}}
$$

\section{Appendix C Derivations section 4}

\section{C.1 Linear quadratic approximation}

The budget restriction 2.3 can be written as

$$
c_{j}+s_{j}^{*}=s_{j}+w_{j}
$$

with $s_{j}^{*}=\sum_{h} s_{h j}^{*}$. Write equity return as $r_{s j}=r_{f}+e_{s j}$. This implies for financial wealth

$$
s_{j}=\frac{r_{f}}{\zeta_{j}} s_{j-1}^{*}+\frac{e_{s j}}{\zeta_{j}} s_{s j-1}^{*}
$$

\footnotetext{
${ }^{9}$ The other root does not give a solution within the admissible range.
} 
Substitute C.2 into C.1 and substitute subsequently equation 2.10 This results in budget equation 4.2. Define function $f$, the instanteneous utility function in 4.1, which has to be linearized

$$
\begin{aligned}
-f\left(\widetilde{\mathbf{z}}_{j}\right) & =\frac{\alpha_{j}}{1-\gamma} c_{j}^{1-\gamma} \\
c_{j} & =\frac{r_{f}}{\zeta_{j}} s_{j-1}^{*}+\frac{e_{s j}}{\zeta_{j}} a r_{f}\left[s_{j-1}^{*}+h_{j-1}^{c}\right]+w_{j}-s_{j}^{*} \\
\widetilde{\mathbf{z}}_{j}^{\prime} & \equiv\left[\begin{array}{ll}
\mathbf{x}_{j} & \widetilde{\mathbf{u}}_{j}
\end{array}\right]=\left[\begin{array}{llll}
s_{j-1}^{*} & e_{s j} & 1 & s_{j}^{*}
\end{array}\right]
\end{aligned}
$$

Write the linear restriction in 4.3 as

$$
\left[\begin{array}{c}
s_{j}^{*} \\
e_{s j+1} \\
1
\end{array}\right]=\left[\begin{array}{ccc}
0 & 0 & 0 \\
0 & 0 & \mu_{s} \\
0 & 0 & 1
\end{array}\right]\left[\begin{array}{c}
s_{j-1}^{*} \\
e_{s j} \\
1
\end{array}\right]+\left[\begin{array}{c}
1 \\
0 \\
0
\end{array}\right]\left[s_{j}^{*}\right]+\left[\begin{array}{c}
0 \\
\omega_{s s} \\
0
\end{array}\right]\left[\varepsilon_{s j+1}\right]
$$

or

$$
\mathbf{x}_{j+1}=\widetilde{\mathbf{A}} \mathbf{x}_{j}+\mathbf{B} \widetilde{\mathbf{u}}_{j}+\mathbf{C} \varepsilon_{j+1}
$$

All nonlinearities in problem 4.1 are absorbed into the composite function $f\left(\widetilde{\mathbf{z}}_{j}\right)$. For each age $j$ we can take a second order Taylor series approximation of $f\left(\widetilde{\mathbf{z}}_{j}\right)$ in point $\overline{\mathbf{z}}_{j}$, the solution of the non-stochastic version of the model

$$
\begin{aligned}
\widehat{f}\left(\widetilde{\mathbf{z}}_{j}\right) & =f\left(\overline{\mathbf{z}}_{j}\right)+\left(\widetilde{\mathbf{z}}_{j}-\overline{\mathbf{z}}_{j}\right)^{\prime} \frac{\partial f}{\partial \widetilde{\mathbf{z}}_{j}}+\frac{1}{2}\left(\widetilde{\mathbf{z}}_{j}-\overline{\mathbf{z}}_{j}\right)^{\prime} \frac{\partial^{2} f}{\partial \widetilde{\mathbf{z}}_{j} \partial \widetilde{\mathbf{z}}_{j}^{\prime}}\left(\widetilde{\mathbf{z}}_{j}-\overline{\mathbf{z}}_{j}\right) \\
& \equiv \widetilde{\mathbf{z}}_{j}^{\prime} \mathbf{M}_{j} \widetilde{\mathbf{z}}_{j}
\end{aligned}
$$

The matrix $\mathbf{M}$ equals

$$
\begin{aligned}
\mathbf{M}_{j} & =d\left[f\left(\overline{\mathbf{z}}_{j}\right)-\left(\frac{\partial f}{\partial \mathbf{z}_{j}}\right)^{\prime} \overline{\mathbf{z}}_{j}+\frac{1}{2} \overline{\mathbf{z}}_{j}^{\prime} \frac{\partial^{2} f}{\partial \mathbf{z}_{j} \partial \mathbf{z}_{j}^{\prime}} \overline{\mathbf{z}}\right] \mathbf{d}^{\prime} \\
& +\frac{1}{2}\left(\frac{\partial f}{\partial \mathbf{z}_{j}} \mathbf{d}^{\prime}-\mathbf{d} \overline{\mathbf{z}}_{j}^{\prime} \frac{\partial^{2} f}{\partial \mathbf{z}_{j} \partial \mathbf{z}_{j}^{\prime}}-\frac{\partial^{2} f}{\partial \mathbf{z}_{j} \partial \mathbf{z}_{j}^{\prime}} \overline{\mathbf{z}}_{j} \mathbf{d}^{\prime}+\mathbf{d} \frac{\partial f^{\prime}}{\partial \mathbf{z}_{j}}\right)+\frac{1}{2}\left(\frac{\partial^{2} f}{\partial \mathbf{z}_{j} \partial \mathbf{z}^{\prime}}\right)
\end{aligned}
$$

with $\mathbf{d}$ a vector with all zero's except a 1 in row 3 corresponding to the constant unity in the state vector, so $1=\mathbf{d}^{\prime} \mathbf{z}_{j}=\mathbf{z}_{j}^{\prime} \mathbf{d}$.

Note, the linearization leads to a modified version of the optimal linear regulator problem. In particular, cross products occur between state and control variables. Hansen and Sargent (2004) discusses a transformation to write the problem in the standard format (equations 4.4 4.5). More precisely, the instantaneous utility function can be written as

$$
\begin{aligned}
\widetilde{\mathbf{z}}_{j}^{\prime} \mathbf{M}_{j} \widetilde{\mathbf{z}}_{j} & =\left[\begin{array}{cc}
\mathbf{x}_{j}^{\prime} & \widetilde{\mathbf{u}}_{j}^{\prime}
\end{array}\right]\left[\begin{array}{cc}
\widetilde{\mathbf{R}}_{j} & \mathbf{W}_{j} \\
\mathbf{W}_{j}^{\prime} & \mathbf{Q}_{j}
\end{array}\right]\left[\begin{array}{c}
\mathbf{x}_{j} \\
\widetilde{\mathbf{u}}_{j}
\end{array}\right] \\
& =\mathbf{x}_{j}^{\prime} \widetilde{\mathbf{R}}_{j} \mathbf{x}_{j}+\widetilde{\mathbf{u}}_{j}^{\prime} \mathbf{Q}_{j} \widetilde{\mathbf{u}}_{j}+2 \widetilde{\mathbf{u}}_{j}^{\prime} \mathbf{W}_{j}^{\prime} \mathbf{x}_{j} \\
& =\mathbf{u}_{j}^{\prime} \mathbf{Q}_{j} \mathbf{u}_{j}+\mathbf{x}_{j} \mathbf{R}_{j} \mathbf{x}_{j}
\end{aligned}
$$


using the transformations

$$
\mathbf{u}_{j}=\widetilde{\mathbf{u}}_{j}+\mathbf{Q}_{j}^{-1} \mathbf{W}_{j}^{\prime} \mathbf{x}_{j}
$$

$$
\mathbf{R}_{j}=\widetilde{\mathbf{R}}_{j}-\mathbf{W}_{j} \mathbf{Q}_{j}^{-1} \mathbf{W}_{j}^{\prime}
$$

Substitute equation C.9 into C.5 leads to transition rule 4.5 if

$$
\mathbf{A}_{j}=\widetilde{\mathbf{A}}-\mathbf{B} \mathbf{Q}_{j}^{-1} \mathbf{W}_{j}^{\prime}
$$

Note, there are no cross productsbetween states and controls for $j=j e$. This implies that a transformation is not necessary for the end year.

\section{C.2 Derivations robust control}

Assume $V=-\mathbf{x}^{\prime} \mathbf{P} \mathbf{x}-d$ for the value function. The construction of the worstcase scenario reads as

$$
{ }_{\mathbf{a}_{j+1}}^{\min } E_{j}\left[\theta \delta^{-1} \zeta_{j+1} \mathbf{a}_{j+1}^{\prime} \mathbf{a}_{j+1}-\delta^{-1} \zeta_{j+1} \mathbf{x}_{j+1}^{\prime} \mathbf{P}_{j+1} \mathbf{x}_{j+1}\right]-\delta^{-1} \zeta_{j+1} d_{j+1}
$$

The first order condition is

$$
\begin{aligned}
\mathbf{a}_{j+1} & =\mathbf{K}_{j} \mathbf{x}_{j} \\
\mathbf{K}_{j} & =\frac{1}{\theta}\left[\mathbf{I}-\frac{1}{\theta} \mathbf{C}^{\prime} \mathbf{P}_{j+1} \mathbf{C}\right]^{-1} \mathbf{C}^{\prime} \mathbf{P}_{j+1}\left[\mathbf{A}_{j}-\mathbf{B} \mathbf{F}_{j}\right]
\end{aligned}
$$

if $\mathbf{u}_{j}=-\mathbf{F}_{j} \mathbf{x}_{j}$. In that case holds $\mathbf{A}_{j} \mathbf{x}_{j}+\mathbf{B} \mathbf{u}_{j}=\mathbf{A}_{j} \mathbf{x}_{j}-\mathbf{B} \mathbf{F}_{\mathbf{j}} \mathbf{x}_{j} \equiv \breve{A}_{j} \mathbf{x}_{j}$ The minimized value of problem C.12 is

$$
-\delta^{-1} \zeta_{j+1} \mathbf{x}_{j}^{\prime} \breve{A}_{j}^{\prime} \mathbf{D}\left(\mathbf{P}_{j+1}\right) \breve{A}_{j} \mathbf{x}_{j}-\delta^{-1} \zeta_{j+1} \operatorname{tr} \mathbf{P}_{j+1} \mathbf{C} \mathbf{C}^{\prime}-\delta^{-1} \zeta_{j+1} d_{j+1}
$$

with

$$
\mathbf{D}\left(\mathbf{P}_{j+1}\right)=\left(\frac{1}{\theta} \mathbf{P}_{j+1} \mathbf{C}\left[\mathbf{I}-\frac{1}{\theta} \mathbf{C}^{\prime} \mathbf{P}_{j+1} \mathbf{C}\right]^{-1} \mathbf{C}^{\prime} \mathbf{P}_{j+1}+\mathbf{P}_{j+1}\right)
$$

Substitution into the objective function 4.10 gives

$$
\begin{aligned}
V_{j}\left(\mathbf{x}_{j}\right)=\max _{\mathbf{u}_{j}} & {\left[-\mathbf{x}_{j}^{\prime} \mathbf{R}_{j} \mathbf{x}_{j}-\mathbf{u}_{j}^{\prime} \mathbf{Q}_{j} \mathbf{u}_{j}\right.} \\
& \left.\quad-\delta^{-1} \zeta_{j+1}\left(\left(\mathbf{x}_{j}^{\prime} \mathbf{A}_{j}^{\prime}+\mathbf{u}_{j}^{\prime} \mathbf{B}^{\prime}\right) \mathbf{D}\left(\mathbf{P}_{j+1}\right)\left(\mathbf{A}_{j} \mathbf{x}_{j}+\mathbf{B} \mathbf{u}_{j}\right)+\operatorname{tr} \mathbf{P}_{j+1} \mathbf{C} \mathbf{C}^{\prime}+d_{j+1}\right)\right]
\end{aligned}
$$


The first order condition is

$$
\mathbf{u}_{j}=-\delta^{-1} \zeta_{j+1}\left[\mathbf{Q}_{j}+\delta^{-1} \zeta_{j+1} \mathbf{B}^{\prime} \mathbf{D}\left(\mathbf{P}_{j+1}\right) \mathbf{B}\right]^{-1} \mathbf{B}^{\prime} \mathbf{D}\left(\mathbf{P}_{j+1}\right) \mathbf{A}_{j} \mathbf{x}_{j} \equiv-\mathbf{F}_{j} \mathbf{x}_{j}
$$

Substitute this result into C.16 and replace the left hand side with the initial guess for $V_{j}$ to get recursive expressions for $\mathbf{P}$ and $d$

$$
\begin{aligned}
\mathbf{P}_{j} & =\mathbf{R}_{j}+\delta^{-1} \zeta_{j+1} \mathbf{A}_{j}^{\prime} \mathbf{D}\left(\mathbf{P}_{j+1}\right) \mathbf{A}_{j}-\delta^{-1} \zeta_{j+1} \mathbf{F}_{j}^{\prime} \mathbf{B}^{\prime} \mathbf{D}\left(\mathbf{P}_{j+1}\right) \mathbf{A}_{j} \\
\mathbf{P}_{j e} & =\mathbf{R}_{j e} \\
d_{j} & =\delta^{-1} \zeta_{j+1}\left(\operatorname{tr} \mathbf{P}_{j+1} \mathbf{C} \mathbf{C}^{\prime}+d_{j+1}\right) \\
d_{j e} & =0
\end{aligned}
$$




\section{References}

Benigno, P. and M. Woodford, 2006, Linear-quadratic approximation of optimal policy problems, Working Paper 12672, National Bureau of Economic Research.

Bovenberg, L., T. Nijman, C. Teulings, and R. Koijen, 2007, Saving and investment over the life cycle: the role of individual and collective pensions, Technical report, Netspar.

De Jong, A., 2005, Bevolkingsprognose 2004-2050: maximaal 17 miljoen inwoners, Bevolkingstrends, Statistisch kwartaalblad over de demografie van Nederland, 53(1):12-18.

Hansen, L. and T. Sargent, 2004, Recursive Models of Dynamic Linear Economies, The MIT Press, Cambridge, Massachusetts.

Hansen, L. and T. Sargent, 2007, Robustness, Princeton university press.

Judd, K., 1999, Numerical methods in economics, The MIT Press, Cambridge, MA.

Kimball, M. S., 1990, Precautionary saving in the small and in the large, Econometrica, 58(1):53-73.

Tallarini, T. D., 2000, Risk-sensitive real business cycles, Journal of Monetary Economics, 45(3):507-532.

Viceira, L. M., 2001, Optimal portfolio choice for long-horizon investors with nontradable labor income, The Journal of Finance, 56(2):433-470. 